text. The prime denotes differentiation with respect to $\zeta$ :

$$
\begin{gathered}
U_{0}^{\prime} / U_{0}=1 /\left(1-\zeta^{2} t^{2}\right) \\
\left(U_{0}^{\prime} / U_{0}\right)^{\prime}=2 \zeta t /\left(1-\zeta^{2} t^{2}\right)^{2} \\
U^{\prime}=\left(U+\frac{1}{4}\right) \zeta^{-1}\left[\frac{1}{\left(1-\zeta^{2}\right)^{\frac{1}{2}}}-\frac{1-\zeta}{1+\zeta}\right] \\
\left(\frac{U^{\prime}}{U}\right)^{\prime}=-(4 U+1)^{-1}\left(\frac{U^{\prime}}{U}\right)^{2}-\zeta^{-1}\left(\frac{U^{\prime}}{U}\right) \\
+\frac{U+\frac{1}{4}}{U}\left[\frac{1}{\left(1-\zeta^{2}\right)^{\frac{1}{2}}}+\frac{2 \zeta}{(1+\zeta)^{2}}\right] \\
\left(V^{\prime} / V\right)^{\prime}=(2 s \zeta-1) / \zeta^{2}(1-s \zeta)^{2}
\end{gathered}
$$

$(U V)^{\prime}=U V\left[\left(U^{\prime} / U\right)+\left(V^{\prime} / V\right)\right]$,$$
(U V)^{\prime \prime}=U V\left[\left(\frac{U^{\prime}}{U}+\frac{V^{\prime}}{V}\right)^{2}+\left(\frac{U^{\prime}}{U}\right)^{\prime}+\left(\frac{V^{\prime}}{V}\right)^{\prime}\right],
$$$$
\phi^{\prime \prime}(\zeta)=\frac{n}{\zeta^{2}}+2\left(\frac{U_{0}^{\prime}}{U_{0}}\right)^{\prime}+\left(\frac{V^{\prime}}{V}\right)^{\prime}+\frac{(U V)^{\prime \prime}}{1-U V}+\left[\frac{(U V)^{\prime}}{1-U V}\right]^{2} .
$$

The saddle-point equation, at $\zeta=\zeta_{0}$ is

$$
\begin{gathered}
s \zeta\left(n-2 \zeta \frac{U_{0}^{\prime}}{U_{0}}+g^{2} \zeta \frac{U^{\prime}}{1+g^{2} U}\right)=\frac{n-2 \zeta U_{0}^{\prime} / U_{0}-1}{1+g^{2} U}, \\
\langle r\rangle=1 /\left[1-U\left(\zeta_{0}\right) V\left(\zeta_{0}\right)\right], \\
n \theta=1 /\left[1-U\left(\zeta_{0}\right) V\left(\zeta_{0}\right)\right]\left(1-s \zeta_{0}\right), \\
\left\langle d_{0}\right\rangle=\zeta_{0} /\left(1-\zeta_{0}^{2}\right) .
\end{gathered}
$$

VOLUME 43, NUMBER 5

1 S E P T EM B E R 1965

\title{
Generalization of Activated-Complex Theory. III. Vibrational Adiabaticity, Separation of Variables, and a Connection with Analytical Mechanics*
}

\author{
R. A. Marcus \\ Noyes Chemical Laboratory, University of Illinois, Urbana, Illinois
}

(Received 12 May 1965)

\begin{abstract}
In Part I, activated-complex theory was extended by including the possibility of a curvilinear reaction coordinate. A separation-of-variables approximation was made in the neighborhood of the activated-complex region of configuration space. In the present paper a more general yet simpler derivation of the final equation is given. It permits subsequent introduction of analytical mechanics in the above neighborhood in a variety of ways such as separation of variables, vibrational adiabaticity, or a method combining certain features of both, the separable-adiabatic approximation. The relationship of these methods is discussed.

Some numerical quantum- and classical-mechanical results obtained for transmission coefficients of nonrotating atom-transfer reactions (linear complexes), using computers, are interpreted in terms of an adiabatic approximation with reasonable agreement. Attention is also called to a modified WBK expression for the transmission coefficient, which generalizes the usual WBK formula in a simple way.
\end{abstract}

\section{INTRODUCTION}

$\mathbf{I}^{\mathrm{N}}$ $\mathrm{N}$ earlier papers of this series, activated-complex theory was extended so as to include a curvilinear reaction coordinate, using quantum mechanics (Part $\mathrm{I})^{1}$ and classical mechanics (Part II). ${ }^{2}$ In the former ${ }^{1}$ the reaction coordinate was assumed to be separable from the other coordinates in a region of configuration space surrounding configurations constituting the activated complex. An internal centrifugal effect on motion along the reaction coordinate was found: When the system could surmount the barrier, the centrifugal effect had a simple classical counterpart. When the system had to tunnel through the barrier, the centrifugal effect was a nonclassical one, negative in nature. ${ }^{1}$

\footnotetext{
* Supported by a grant from the National Science Foundation.

1 R. A. Marcus, J. Chem. Phys. 41, 2614 (1964).

2 R. A. Marcus, J. Chem. Phys. 41, 2624 (1964).
}

An extended ${ }^{3}$ Stäckel-Robertson formalism was introduced to make the separation of variables. One of the several mathematically equivalent final expressions deduced for the rate constant contained no "Stäckel coefficients." It was perhaps the most useful one and is derived here without assuming separation of variables. In applications to actual rate problems one is then free to introduce a separation-of-variables assumption or, instead, an assumption that all motions except the one along the reaction coordinate are "adiabatic." Two ways of introducing an adiabatic assumption of this nature are described. One of these, based on the above work on the extended Stäckel-Robertson formalism, ${ }^{3}$ includes separation of variables as a special case and has a certain useful physical interpretation.

\footnotetext{
${ }^{3}$ R. A. Marcus, J. Chem. Phys. 41, 603 (1964). There is a typographical error in Eq. (25), where the $\chi_{N}{ }^{1}$ in the denominator should be replaced by $\chi_{N}{ }^{t}$.
} 
A major aim of the present paper and that of Part I is to introduce analytical mechanics for motion in the activated-complex neighborhood of configuration space. The connection between this work and two or three recent numerical computations made with electronic computers is described.

In another section of the paper transmission coefficients are discussed for curvilinear coordinate systems. ${ }^{3 \mathrm{a}}$ Attention is called to a recent approximate phaseintegral expression for transmission coefficients, ${ }^{4}$ which has apparently not yet been applied to problems in chemical physics. It generalizes the usual WBK expression in a simple way.

\section{REACTION RATE CONSTANT}

The Schrödinger equation in curvilinear coordinates $q^{1}$ to $q^{n}$ has the well-known form (1), where $1 / g$ is the determinant of the $g^{i j}$ 's, as in Part $I$, and $U$ is the potential-energy function:

with

$$
H \psi=E \psi,
$$

$$
H=-\frac{\hbar^{2}}{2} \sum_{i, j=1}^{n} \frac{1}{g^{3}} \frac{\partial}{\partial q^{i}} g^{\frac{1}{2}} g^{i j} \frac{\partial}{\partial q^{j}}+U\left(q^{1}, \cdots, q^{n}\right) .
$$

We assume that there is a hypersurface in the $n$ dimensional space such that passage across it leads to reaction. The set of points in configuration space defining this hypersurface is the activated complex. When the dynamically appropriate coordinates are Cartesian the position of this hypersurface does not depend on the approximate or exact constants of the motion. It can depend on them when the appropriate coordinates are curvilinear. ${ }^{1}$

Coriolis effects will be neglected [i.e., cross terms between vibrational and rotational angular momenta in (2) are neglected]. The internal motion of the system then occurs in a potential field which contains centrifugal effects. The latter affect the position of the hypersurface.

It will be supposed that some distance from this hypersurface, closer to the "reactants' region" of configuration space, in a small interval the motion along the reaction coordinate can be described classically and the probability of finding a system moving in that interval in the forward direction (i.e., towards products) has approximately the equilibrium value. Diffraction effects for motion along the reaction coordinate are presumed to occur principally in the immediate activated complex neighborhood. There, the wavelength for forward motion is longer and, in addition, tunneling may occur. The reaction coordinate, denoted by $q^{r}$ in Parts I and II, is denoted by $q^{1}$ here.

3a Note added in proof: Interesting calculations of transmission coefficients have been discribed by $\mathrm{J}$. $O$. Hirschfelder and coworkers for certain potential-energy surfaces having infinitely steep walls encompassing channels in which the potential energy is constant [J. Chem. Phys. 11, 276 (1943) ; 30, 1032 (1959)].

${ }^{4} \mathrm{~J}$. Heading, An Introduction to Phase Integral Methods (Methuen and Company Ltd., London, 1961), Chap. 5.
The reaction coordinate leading from the cited interval, through activated complex, to products is to be chosen in a way which facilitates solution of the dynamical equations approximately or exactly in the neighborhood of the activated complex.

Any $q^{1}$-coordinate hypersurface in the above classical $q^{1}$ interval is singled out and denoted by $S$. In this region the wavefunction $\psi$ is written as

$$
\psi=\exp \left(i S_{1} / \hbar\right) \Psi_{2},
$$

where $S_{1}$ depends only on $q^{1}$ and where $\Psi_{2}$ is either independent of $q^{1}$ or depends only weakly on it there. Introduction of (3) into (1), neglect of the appropriate powers of $\hbar$ and of the derivatives of $\Psi_{2}$ with respect to $q^{1}$ yields, for classical $q^{1}$ region only,

$$
\left(-\frac{\hbar^{2}}{2} \sum_{i, j \neq 1} \frac{1}{g^{\frac{1}{2}}} \frac{\partial}{\partial q^{2}} g^{\frac{1}{2}} g^{i j} \frac{\partial}{\partial q^{j}}+U\right) \Psi_{2}=\left(E-\frac{1}{2} g^{11} p_{1}^{2}\right) \Psi_{2},
$$

where $p_{1}$, which equals $d S_{1} / d q^{1}$, is the classical momentum conjugate to $q^{1}$. $\Psi_{2}$ was denoted by $\psi^{\prime}$ in Part I and was assumed there to be independent of $q^{1}$ (separation-of-variables approximation).

For convenience of counting quantum states, the entire system is enclosed in a finite volume so that $\Psi_{2}$ has discrete eigenvalues, characterized by a quantum number $\lambda$. Because of the equilibrium postulate, the probability $P d p_{1} \prod_{i} d q^{i}$ of finding a system simultaneously in a range of coordinates $\Pi_{i} d q^{i}$ near the hypersurface $S$, in momentum range $p_{1}, p_{1}+d p_{1}\left(p_{1} \geq 0\right)$, and in a quantum state $\lambda$, has the equilibrium value (5), as in Part $\mathrm{I}^{5}$ :

$$
\begin{aligned}
& P d p_{1} \prod_{i} d q^{i} \\
& \quad=e^{-E / k T}\left|\Psi_{2}\right|{ }^{2} g^{\frac{1}{2}}\left(\prod_{i} d q^{i}\right) d p_{1} /\left(h Q \int\left|\Psi_{2}\right|^{2} g^{\frac{1}{2}} \prod_{i \neq 1} d q^{i}\right),
\end{aligned}
$$

where $E$ is the energy appearing in (4) and $Q$ is the partition function of the reactants.

The simultaneous chance of finding the system in the volume element $g^{\frac{1}{3}} \prod_{i} d q^{i}$, in the state $\lambda$ and in an energy interval $d E$ is now obtained by replacing $d p_{1}$ by $\left(\partial p_{1} / \partial E\right)_{\lambda} d E$. The corresponding probability term per unit interval of $q^{1}$ is obtained by dividing by $d q^{1}$. The contribution of these states to the system's crossing of the hypersurface $S$ equals this expression multiplied by $\dot{q}^{1}$. The transmission coefficient for this value of $\lambda$ and of $E$, i.e., the probability that a system crossing the hypersurface $S$ in the forward direction yields products, is denoted by $\kappa(\lambda, E)$. The reaction

5 The equilibrium probability of finding the system in a particular state $\lambda$ and in the small-phase space volume element $\Delta p_{1} \Delta q^{1}$ is $\exp (-E / k T) \Delta p_{1} \Delta q^{1} / h Q$, since there are $\Delta p_{1} \Delta q^{1 / h}$ quantum states in this volume element. In any one of these states the probability of finding the system in the configuration space volume element of $g^{3} \Pi_{i} d q^{i}$ is $\left|\Psi_{2}\right|^{2} g^{3} \Pi_{i} d q^{i} / \int\left|\Psi_{2}\right|^{2} g^{i} \Pi_{i} d q^{i}$, the integration over $q^{1}$ being only over the very small interval $\Delta q^{1}$ Multiplication of these two probabilities and replacing $\Delta$ 's by $d$ 's yields (5). 
rate constant is then obtained by multiplying the previous expression by $\kappa$ and integrating, as in

$$
k_{\text {rate }}=\frac{k T}{h} \sum_{\lambda} \int_{E} \kappa(\lambda, E) \int_{\mathbf{q}^{2}} \dot{q}^{[}\left[e^{-E / k T}\left|\Psi_{2}\right|^{2} g^{\frac{2}{2}}\left(\frac{\partial p_{1}}{\partial E}\right)_{\lambda} d \mathbf{q}^{2} /\left(Q \int\left|\Psi_{2}\right|^{2} g^{\frac{1}{2}} d \mathbf{q}^{2}\right)\right] \frac{d E}{k T},
$$

where we have used an abbreviation,

$$
d \mathbf{q}^{2}=\prod_{i \neq 1} d q^{i}
$$

We now suppose that the coordinate system is an orthogonal one. The value of $\dot{q}^{1}$ is then $g^{11} p_{1}$. The derivative $\left(\partial p_{1} / \partial E\right)_{\lambda}$ is obtained by calculating the variation $\delta p_{1}$ arising from a $\delta E$ at fixed $\lambda$ and $q^{\mathbf{1}}$. Any corresponding variation in $\Psi_{2}$ is subject to

$$
\int\left|\Psi_{2}\right|^{2} g^{\frac{2}{2}} d \mathbf{q}^{2}=\text { constant. }
$$

The differential operator in (4) will be denoted by $T^{\prime}$. On performing the variation we obtain

$$
\left(T^{\prime}+U\right) \delta \Psi_{2}=\left(\delta E-g^{11} p_{1} \delta p_{1}\right) \Psi_{2}
$$

subject to

$$
\operatorname{Re} \int \Psi_{2}{ }^{*} \delta \Psi_{2} g^{\frac{1}{2}} d \mathbf{q}^{2}=0,
$$

where Re denotes "real part of."

The following relation is immediately verified upon integration by parts.

$$
\int\left(\Psi_{2}{ }^{*} T^{\prime} \delta \Psi_{2}\right) g^{\frac{3}{3}} d \mathbf{q}^{2}=\int\left(\delta \Psi_{2} T^{\prime} \Psi_{2}{ }^{*}\right) g^{\frac{1}{3}} d \mathbf{q}^{2} .
$$

Multiplication of (9) by $\Psi_{2}{ }^{*} g^{\frac{1}{2}} d \mathbf{q}^{2}$, integration, taking the real part of the resulting equation, and use of (10) and (11) yields

$$
\text { (at fixed } \lambda \text { and } q^{1} \text { ) } \delta E=p_{1} \delta p_{1}\left\langle g^{11}\right\rangle
$$

where \langle\rangle is defined as

$$
\langle f\rangle=\int f\left|\Psi_{2}\right|^{2} g^{\frac{1}{2}} d \mathbf{q}^{2} /\left(\int\left|\Psi_{2}\right|^{2} g^{\frac{1}{2}} d \mathbf{q}^{2}\right) .
$$

Equation (14) then follows:

$$
k_{\text {rate }}=\frac{k T}{h Q} \sum_{\lambda} \int_{k} \kappa(\lambda, E) e^{-E / k T} \frac{d E}{k T} .
$$

It is convenient to measure $U$ relative to its value $U_{0}$ at some point on or near the activated-complex hypersurface (for example, the point can be the saddle point if there is one) and to measure $E$ relative to that $U_{0}$, rather than measuring both relative to the potential energy of the stablest configuration of the reactants, $U_{0}^{\text {react }}$. If $E$ is now measured relative to $U_{0}$ we obtain from (14) an equation identical with Eq. (22) in Part I:

$$
k_{\text {rate }}=\frac{k T}{h Q} \sum_{\lambda} \int_{E} \kappa(\lambda, E) e^{-E / k T} e^{-\Delta U / k T} \frac{d E}{k T},
$$

where $\Delta U$ denotes $U_{0}-U_{0}$ react.
If the $q^{1}$ motion were treated classically at the activated-complex hypersurface, $\kappa(\lambda, E)$ would have a very simple form: It vanishes for $E$ less than some quantity $\epsilon_{\lambda}$ and equals unity for $E$ 's in excess of $\epsilon_{\lambda}$. This $\epsilon_{\lambda}$ equals the value of $E$ below which the momentum $p_{1}$ has an imaginary value somewhere in the activated-complex neighborhood. One obtains

(classical $\left.q^{1}\right) \quad k_{\text {rate }}=(k T / h)\left(Q_{c} / Q\right) \exp (-\Delta U / k T)$,

where

$$
Q_{c}=\sum_{\lambda} \exp \left(-\epsilon_{\lambda} / k T\right) .
$$

We consider several calculations for $\kappa(\lambda, E)$ and $\epsilon_{\lambda}$ in later sections.

An equation related to (15) may be derived in other ways, with different approximations. For example, one such assumption is that the motion of the totality of coordinates $\mathbf{q}^{2}$ is adiabatic throughout the region from reactants to products and not merely in the activated complex neighborhood. ${ }^{6}$ It then suffices to assume that the distribution of the reactants' configurations is an equilibrium one. Such assumptions may be compared with those used here.

The assumption of equilibrium for the configurational distribution of the reactants plus the assumption of adiabaticity for $\mathbf{q}^{2}$ motion throughout imply that the distribution of systems at $S$ moving in a forward direction is indeed an equilibrium one. i.e., they would justify an assumption used here. However, the latter equilibrium condition can be achieved under a milder assumption (adiabaticity from reactants' region to hypersurface $S$ ), and can be achieved approximately under still milder assumptions.

\section{REACTION-PATH DEGENERACY}

In the derivation of Eq. (16), we tacitly considered the formation of the activated complex by way of some distinguishable reaction path from any given isomer of the reactants. There may be one or more such distinguishable paths from the given isomer. The sequence of intermediate structures in each of these paths is either a "geometrical isomer" or an "optical isomer" of the sequence in any other path proceeding from the same isomer. (These remarks apply even if the reactants themselves have no isomers.)

Let there be $\gamma$ optically isomeric reaction paths for a particular geometrically isomeric path from the given isomer of the reactants. Each optically isomeric path

\footnotetext{
${ }^{6}$ In a very interesting paper in Z. Naturforsch. 18a, 607 (1963) L. Hofacker has derived an equation related to (14) by making this assumption. He also presents a formalism for including nonadiabatic corrections, at least when these corrections are small.
} 
makes an equal contribution to the rate, so the contribution of these $\gamma$ paths to the rate is $\gamma$ multiplied by (16). The total rate constant is then obtained by summation over all geometrically isomeric paths from the given isomer of reactants:

$$
k_{\text {rate }}{ }^{\text {total }}=\sum_{\substack{\text { geometrically } \\ \text { isomeric } \\ \text { paths }}}\left(\gamma k_{\text {rate }}\right) .
$$

The value of $k_{\text {rate }}$ differs for paths that are "geometrical isomers" of each other.

These results can be applied to a discussion of statistical factors in reaction rates.

\section{SEPARATION OF VARIABLES APPROXIMATION FOR DETERMINATION OF $\kappa$ AND $\epsilon_{\lambda}$}

It has been shown ${ }^{7}$ that in the immediate vicinity of a saddle point of a potential-energy surface, curvilinear coordinates may be introduced which permit separation of variables. The region of validity is larger than that for the standard harmonic expansion of the potential-energy function near a saddle point, and the analysis has provided some dynamical insight. However, numerical investigations are needed to determine whether the region of validity is large enough for present usage. A more general formulation (separableadiabatic) is described in a later section.

To calculate $\kappa$ and $\epsilon_{\lambda}$ we first recall the conditions in the extended Stäckel-Robertson formalism for a separation of variables into $m$ sets. To express these conditions we re-enumerate the variables, letting the number in the $\mu$ th set be $h_{\mu}$ and designating them as $q^{\mu_{i}}$ with $i=1$ to $h_{\mu}$. Here, $m$ can equal 2 ( $q^{1}$ in one set and the remaining $q^{i}$ 's in a second set) or $m$ can exceed 2 if one wishes.

\section{Conditions on the Kinetic-Energy Operator}

It is stipulated that there exist functions $\phi_{\mu \nu}$ of the $\mu$ th set of coordinates, with determinant $\phi$ and with conjugate quantities $\phi^{\mu \nu}$,

$$
\sum_{\mu=1}^{m} \phi^{\mu \nu} \phi_{\mu \lambda}=\delta_{\lambda}{ }^{\nu}
$$

such that the following three conditions are fulfilled:

$$
\begin{aligned}
& g^{\frac{1}{2}}=\phi \prod_{\mu=1}^{m} f_{\mu}, \\
& \left(\operatorname{det}^{h_{\mu} g \dot{\mu i \mu j}}\right)^{1 / h_{\mu}}=\phi^{\mu 1}, \\
& g^{\mu_{i} \mu_{j}}=f^{\mu_{i} \mu_{i}} \phi^{\mu_{1}} / f_{\mu},
\end{aligned}
$$

where $f^{\mu_{i} \mu_{i}}$ and $f_{\mu}$ are functions of the $\mu$ th set of coordinates only, and where it follows from (20) and (21) that $f_{\mu}$ equals

$$
\underset{i, j=1}{\left(\operatorname{det}^{h_{\mu}} \mu_{i} \mu_{j}\right)^{1 / h_{\mu}} .}
$$

\footnotetext{
7 R. A. Marcus, J. Chem. Phys. 41, 610 (1964).
}

\section{Condition on the Potential Energy}

$$
U=\sum_{\mu=1}^{m} \phi^{\mu_{1}} X_{\mu}
$$

where $X_{\mu}$ is a function of the coordinates of the $\mu$ th set only.

In this formalism the equation for $p_{1}$ is given by Eq. (A4) of Part I:

$$
\frac{1}{2} g^{11} p_{1}^{2}=\sum_{\nu=1}^{m} \alpha_{\nu} \phi_{1 v}-X_{1}
$$

where $\alpha_{1}=E$ and the remaining $\alpha_{\nu}$ are separation constants.

Equation (4) for $\Psi_{2}$ (freed from $q^{1}$ as described below) is solved for $\Psi_{2}$. One obtains thereby a dependence of the $\alpha_{v}$ on $E$ for the given $\lambda$. The righthand side of (23) now depends only on $q^{1}, \lambda$, and $E$. For $E$ less than some quantity $\epsilon_{\lambda}, p_{1}$ given by (23) is imaginary for some $q^{1}$ in the activated complex region of configuration space. Thereby, the $\epsilon_{\lambda}$ in (17) can be determined.

The wavefunction in this separation-of-variables approximation is given by

where

$$
\psi=\psi_{1}\left(q^{1}\right) \Psi_{2}\left(\mathbf{q}^{2}\right),
$$

$$
\Psi_{2}=\prod_{\mu=2}^{m} \psi_{\mu},
$$

$\psi_{\mu}$ being the wavefunction for the $\mu$ th set of coordinates.

To obtain $\kappa(\lambda, E)$ it is necessary to first solve the $\Psi_{2}$ equation, (4). Introduction of the conditions permitting separation of variables converts Eq. (4) to the following equations:

$$
\mu=2, \cdots, m \quad\left(T_{\mu}+X_{\mu}\right) \psi_{\mu}=\sum_{\nu=1}^{m} \alpha_{\nu} \phi_{\mu \nu} \psi_{\mu}
$$

where

$$
T_{\mu}=-\left(\hbar^{2} / 2 f_{\mu}\right) \sum_{i, j}\left(\partial / \partial q^{i}\right) f^{\mu_{i} \mu_{i}}\left(\partial / \partial q^{j}\right) .
$$

Solution of Eqs. (25) yields the $\alpha_{\nu}$ as a function of $E$ for the given $\lambda$. This result is then introduced into

${ }^{8}$ On using Eqs. (19) to (21), $g^{\ddagger} g^{\mu i \mu j}$ can be rewritten as $\phi \phi^{\mu 1} f^{\mu i \mu j} \Pi_{y, f_{y}}$. Since $\phi \phi^{\mu 1}$ is the cofactor of $\phi_{\mu 1}$ in the determinant of the $\phi_{\mu}$ 's, it does not contain the $\mu$ th row, and so is independent of the coordinates in the $\mu$ th set. $\Pi_{o \neq \mu} f$, is also independent of these coordinates. Both factors can be extracted from the differential operator in (4). Use of Eq. (19) again then converts this first term in (4) to $\Sigma_{\mu \neq \neq 1} \phi^{\mu 1} T_{\mu}$. Use of Eq. (22) then leads from (4) to

$$
\sum_{\mu=1}^{m} \phi^{\mu 1}\left(\tau_{\mu}+X_{\mu}\right)=\alpha_{1}
$$

where $\tau_{1}=p_{1}^{2} / 2$ and $\tau_{\mu}=T_{\mu}$ otherwise. On using (18) one then finds

$$
\sum_{\mu=1}^{m} \phi^{\mu 1}\left(\tau_{\mu}+X_{\mu}-\sum_{\nu=1}^{n} \alpha_{\nu} \phi_{\mu \nu}\right)=0 \text {. }
$$

Equation (25) is consistent with this result. However, Eq. (25) may be derived from it by using the linear-vector-space arguments used to derive Eq. (14) of Ref. 3 from Eq. (11) there. 
the equation for $\psi_{1}$. In this formalism ${ }^{3}$ the $\psi_{1}$ equation is given by

$$
\left(T_{1}+X_{1}\right) \psi_{1}=\sum_{\nu=1}^{m_{2}} \alpha_{\nu} \phi_{1 \nu} \psi_{1}
$$

where

$$
T_{1}=-\frac{\hbar^{2}}{2} \frac{1}{f_{1}} \frac{\partial}{\partial q_{1}} f_{1} \frac{\partial}{\partial q^{1}} .
$$

Equation (27) is the quantum counterpart of (23). Solution of Eq. (27) yields a value for the transmission coefficient $\kappa$ as a function of $E$ and $\lambda^{1,3}$

The Cartesian approximation to these equations is described in Part I. It yields the usual activated-complex-theory formula in a straightforward manner. Application of (25) and (27) to several curvilinear coordinate systems was also discussed in Part I.

\section{ADIABATIC- $q^{2}$ APPROXIMATION FOR DETERMINATION OF $\kappa$ AND $\epsilon_{\lambda}$}

It is possible that the motion along some coordinate $q^{1}$ leading from reactants to products is not separable over a sufficiently large region in the activated-complex vicinity. In this case the assumption of separability might be replaced by one in which the $\mathbf{q}^{2}$ motion is adiabatic during the motion along $q^{1}$ in this vicinity. The wavefunction $\psi$ then becomes

$$
\psi=\psi_{1}\left(q^{1}\right) \Psi_{2}\left(q^{1}, \mathbf{q}^{2}\right),
$$

where $\Psi_{2}$ now depends weakly on $q^{1}$. In both the separable and adiabatic approximations $\Psi_{2}$ remains in the same quantum state $\lambda$ throughout the motion in this activated-complex neighborhood.

The function $\Psi_{2}$ is determined by solving the following equation, where $T^{\prime}$ is the differential operator in (4),

$$
\left(T^{\prime}+U\right) \Psi_{2}=\eta_{\lambda} \Psi_{2},
$$

thereby determining $\eta_{\lambda}$ for each value of $q^{1}$ of interest. The function $\psi_{1}$ is then determined by introducing (29) into (4), neglecting the derivatives of $\Psi_{2}$ with respect to $q^{1}$, multiplying by $\Psi_{2}{ }^{*} g^{\frac{1}{2}} d \mathbf{q}^{2}$, integrating over $\mathbf{q}^{2}$, and obtaining

$$
-\frac{1}{2} \hbar^{2}\left\langle\left(1 / g^{\frac{1}{1}}\right)\left(\partial / \partial q^{1}\right) g^{\frac{1}{11}} g^{11}\left(\partial / \partial q^{1}\right)\right\rangle \psi_{1}+\eta_{\lambda} \psi_{1}=E \psi_{1},
$$

where \langle\rangle is given by (13). This equation is solved for $\kappa$ for any given $E$ and $\lambda$.

When the $q^{1}$ motion is treated classically by setting $\psi_{1}=\exp \left(i S_{1} / \hbar\right)$ and neglecting appropriate powers of $\hbar$, (31) becomes

$$
\frac{1}{2}\left\langle g^{11}\right\rangle p_{1}^{2}+\eta_{\lambda}=E,
$$

which permits one to determine $\epsilon_{\lambda}$, the value of $E$ below which $p_{1}^{2}$ can be negative.

When the kinetic-energy operator satisfies the separability conditions (19) to (21), Eq. (31) can be simplified. It becomes

$$
-\frac{1}{2} \hbar^{2}\left\langle g^{11}\right\rangle f_{1}^{-1}\left(\partial / \partial q^{1}\right) f_{1}\left(\partial / \partial q^{1}\right) \psi_{1}+\eta_{\lambda} \psi_{1}=E \psi_{1} .
$$

\section{SEPARABLE-ADIABATIC APPROXIMATION FOR DETERMINATION OF $\kappa$ AND $\epsilon_{\lambda}$}

The approach just presented has a dynamical limitation discussed later. We can avoid it for systems of coordinates in which the kinetic-energy operator satisfies the separability conditions (19) to (21). We call the new approach a separable-adiabatic approximation to emphasize the above difference. When these conditions (19) to (21) are satisfied, the Schrödinger equation (1) can be written as

$$
\left(\sum_{\mu=1}^{m} \phi^{\mu} T_{\mu}+U\right) \psi=\alpha_{1} \psi
$$

where $T_{\mu}$ is given by (28) and (26).

We now suppose that $U$ can be written as

$$
U=\phi^{11} X_{1}+\sum_{\mu=2}^{m} \phi^{\mu_{1}} Y_{\mu},
$$

where $X_{1}$ depends on $q^{1}$ alone and $Y_{\mu}$ depends strongly on the coordinates in Set $\mu$ but weakly on $q^{1}$. For any given $q^{1}$ the following equations are solved for each $\psi_{\mu}$ and $\alpha_{\nu}$ as a function of $\alpha_{1}$ and $\lambda$ :

$$
(\mu=2 \text { to } m) \quad\left(T_{\mu}+Y_{\mu}\right) \psi_{\mu}=\sum_{\nu=1}^{m} \alpha_{\nu} \phi_{\mu \nu} \psi_{\mu}
$$

These $\alpha_{\nu}$ 's now depend weakly on $q^{\mathrm{I}}$, in contrast to the separation-of-variables equation (25) where the $\alpha_{\nu}$ 's were constants.

The wavefunction (29) is now introduced into (34), and the derivatives of $\Psi_{2}$ with respect to $q^{1}$ are neglected. Manipulation based on (18) then yields the same result as (26):

$$
\left(T_{1}+X_{1}\right) \psi_{1}=\sum_{\nu=1}^{m} \alpha_{\nu} \phi_{1 \nu} \psi_{1} .
$$

Since each $\alpha_{v}$ is already known as a function of $q^{1}$ for any given $E$ and $\lambda$, from the solution of (36), Eq. (37) can then be solved for $\psi_{1}$ and for $\kappa(\lambda, E) . \epsilon_{\lambda}$ can be determined from the classical counterpart of (37), namely (23), in the same way as before, remembering that $\alpha_{\nu}$ is now a function of $q^{1}$.

In the special case that $Y_{\mu}$ equals $X_{\mu}$, these results reduce to those obtained earlier by the separation-ofvariables approximation.

The weak dependence of $\alpha_{\nu}$ on $q^{1}$, in contrast to the separation-of-variables approximation where $\alpha_{y}$ was a constant, can have an immediate physical interpretation: Suppose, for example, one considers a nonrotating linear-collision complex in a three-center reaction with an activation-energy reaction, $\mathrm{A}+\mathrm{BC} \rightarrow \mathrm{AB}+\mathrm{C}$, and introduces two coordinates $r_{\mathrm{AB}}$ and $r_{\mathrm{BC}}$, making the usual contour diagram of the potential energy, with the kinetic energy in diagonal form. (Skewed axes are used for the two above coordinates, therefore.) The $\boldsymbol{q}^{2}$ motion becomes a single vibrational motion, since we ignore for purposes of the following remarks the 
external translations, the rotations, and the bending vibrations. Polar coordinates satisfy the separability conditions (19) to (21). When they are used over a limited $q^{1}$ interval, such that the vibrational motion is radial and the motion along $q^{1}$ is along the arc of a circle, $\alpha_{2}$ becomes an internal angular momentum except for a multiplicative constant. When the potential energy is constant along the arc and depends only on $q^{2}$, it can be shown to satisfy the separability condition (22), and so $\alpha_{2}$ is indeed a constant. In the actual reacting system the potential energy is not constant along this arc, and so $\alpha_{2}$ varies with $q^{1}$, as in the separable-adiabatic approximation.

\section{RELATION OF SEPARATION-OF-VARIABLES AND ADIABATIC APPROXIMATIONS}

We have already seen that the first of these approximations is a special case of the separable-adiabatic one. It is not a special case of the ordinary adiabatic one, a result established as follows.

If the separation-of-variables approximation were a special case of the ordinary adiabatic one, the former could be obtained from the latter by introducing conditions (19) to (22). Introduction of these conditions for $m=2$ leads to the following deductions from (30) and (33):

$$
\begin{gathered}
\phi^{21}\left(T_{2}+X_{2}\right) \Psi_{2}=\left(\eta_{\lambda}-\phi^{11} X_{1}\right) \Psi_{2}, \\
\left(\left\langle\phi^{11}\right\rangle T_{1}+\eta_{\lambda}\right) \psi_{1}=\alpha_{1} \psi_{1} .
\end{gathered}
$$

Comparison of these equations with the separated ones (25) and (27) for $m=2$ shows the latter to be special cases of the former if

$$
\eta_{\lambda}=\phi^{11} X_{1}+\phi^{21} \sum_{\nu=1}^{2} \alpha_{\nu} \phi_{2 y}
$$

and if

$$
\eta_{\lambda}=\left\langle\phi^{11}\right\rangle X_{1}-\left\langle\phi^{11}\right\rangle \sum_{\nu=1}^{2} \alpha_{\nu} \phi_{1 \nu}+\alpha_{1}
$$

Elimination of $\eta_{\mathrm{\lambda}}$ then yields

$$
\left(\phi^{11}-\left\langle\phi^{11}\right\rangle\right) X_{1}-\left(\phi^{11}-\left\langle\phi^{11}\right\rangle\right) \sum_{\nu=1}^{2} \alpha_{\nu} \phi_{1 \nu}=0
$$

upon using (18). Since $X_{1}$ and the $\phi_{\mu \nu}$ 's are independent, Eq. (42) shows that now $\left\langle\phi^{11}\right\rangle=\phi^{11}$, and thereby that $\phi^{11}$ depends only on $q^{1}$. Equation (41) yields no new restrictions on the $\phi_{\mu \nu}$ 's since it is readily satisfied: All quantities on the right depend only on $q^{1}$ as does $\eta_{\lambda}$. Equation (40) yields no new restriction since it is functionally dependent on (41) and (42).

Thus, the separable approximation is a special case of the ordinary adiabatic one only if $\phi^{11}$ depends on $q^{1}$ alone. This restriction, however, is severe. Since $\phi^{11}$ equals $g^{11}$, the restriction means that the perpendicular distance between successive $q^{1}$-coordinate hypersurfaces is independent of position on the hyper- surface (Ref. 3, p. 609). Since the $q^{1}$ coordinate is orthogonal to the others, diagrams suggest and exact calculations show ${ }^{9}$ that a $q^{1}$ coordinate for which $g^{1 i}=0$ for $i \neq 1$ and $g^{11}=$ independent of $q_{2}$ (and hence for which $g_{1 j}=0$ and $g_{11}=$ independent of $\left.\mathbf{q}_{2}\right)$ is a straight line. The usual reaction paths are curved, so such a $q^{1}$ coordinate is not dynamically suitable to describe approximately separable or adiabatic motion in these systems.

\section{TRANSMISSION COEFFICIENTS IN CURVILINEAR COORDINATE SYSTEMS}

To make use of a WKB-type formula on transmission coefficients it is convenient to rewrite the earlier equations for $\psi_{1}$ by making a change of variable $\psi_{1}=\zeta_{1} / f_{1}{ }^{3}$. One obtains

$$
\left(d^{2} \zeta_{1} / d q^{1^{2}}\right)+\chi_{1} \zeta_{1}=0
$$

where [Eqs. (27), (37)]

$$
\chi_{1}=\frac{2}{\hbar^{2}}\left(\sum_{\nu=1}^{m} \alpha_{\nu} \phi_{1 v}-X_{1}\right)-\frac{1}{4}\left(\frac{d \ln f_{1}}{d q^{1}}\right)^{2}-\frac{1}{2} \frac{d^{2} \ln f_{1}}{d q^{1^{2}}}
$$

and [Eq. (33)]

$$
\chi_{1}=\frac{2}{\hbar^{2}} \frac{\left(E-\eta_{\lambda}\right)}{\left\langle g^{11}\right\rangle}-\frac{1}{4}\left(\frac{d \ln f_{1}}{d q^{1}}\right)^{2}-\frac{1}{2} \frac{d^{2} \ln f_{1}}{d q^{1^{2}}} .
$$

$\psi_{1}$ has an asymptotic behavior

$$
\exp \left( \pm \int i \chi_{1}^{\frac{1}{2}} d q^{1}\right) / \chi_{1}^{\frac{1}{1} f_{1}^{3}}
$$

The boundary conditions and the expression for the transmission coefficient have been given previously. ${ }^{3}$

Transmission coefficients based on a WKB-type solution can be obtained for (43). We do not use the usual WKB expression, which is derived for tunneling only and which also assumes the classical turning points to be far apart. As a result of the latter assumption this usual expression is in error by a factor of 2 when the system just has enough energy to surmount the barrier.

Instead, we use a recent phase-integral expression which takes cognizance of possible close proximity of the turning points and which applies to energies sufficient for surmounting the barrier also. ${ }^{4}$ The classical turning points occur when tunneling occurs. They

\footnotetext{
${ }^{9}$ We utilize equations in A. J. McConnell, Applications of Tensor Analysis (Dover Publications, Inc., New York, 1957): We first remark that if $g^{1 i}=0$ for $i \neq 1$, it can be shown from the relation $\Sigma_{j g^{i j}} g_{j k}=\delta_{k}{ }^{i}$ that $g_{11}=1 / g^{11}$ and $g_{1 i}=0$ for $i \neq 1$. The equations of a straight line are given in terms of intrinsic derivatives in Eq. 13, p. 161 of McConnell's text. On recalling that along a $q^{1}$ coordinate curve only the value of $q^{1}$ is changing tand hence only $d x^{1 / d} d s$ on p. 161 differs from zero and it equals $\left.1 /\left(\mathrm{gu}^{\mathrm{k}}\right)^{k}\right]$ and on evaluating the Christoffel symbols of the type

$$
\left\{\begin{array}{c}
i \\
11
\end{array}\right\}
$$

for the above $g_{i 1}$ 's one verifies that this $q^{1}$-coordinate curve is indeed straight.
} 
represent the real zeros of the function $\chi_{1}$. In this case $\chi_{1}$ was represented as in (46) in the cited reference,

$$
\chi_{1}\left(q^{1}\right)=\left(q^{1^{2}}-a^{2}\right) \beta\left(q^{1}\right),
$$

where $\beta\left(q^{1}\right)$ is a real, positive function of $q^{1}$ along the real axis with no zeros in this neighborhood, and where the origin of $q^{1}$ is chosen so that the zeros of $\chi_{1}$ occur at $\pm a$.

When the system can surmount the barrier the two zeros of $\chi_{1}$ in the neighborhood of the maximum of the barrier are no longer real. $\chi_{1}$ was then written as in (47), with a suitable (new) choice of the origin of $q^{1}$. (An expression was also given in Ref. 4 for the case where the two zeros are not pure imaginary.)

$$
\chi_{1}\left(q^{1}\right)=\left(q^{1^{2}}+b^{2}\right) \Gamma\left(q^{1}\right) .
$$

$\Gamma$ is real and positive on the real axis, with no real or complex zeros in the neighborhood. The following equations were obtained for the transmission coefficient ${ }^{10}$ :

$$
\begin{array}{ll}
\kappa=1 /\left(1+A^{2}\right) & \text { (tunneling), } \\
\kappa=1 /\left(1+B^{2}\right) & \text { (surmounting), }
\end{array}
$$

where

$$
\begin{aligned}
& A=\exp \left[\int_{-a}^{a}\left(-\chi_{1}\right)^{\frac{1}{2}} d q^{1}\right], \\
& B=\exp \left[-\int_{-b}^{b} \chi_{1^{\frac{1}{2}}}(i y) d y\right] .
\end{aligned}
$$

In Eq. (51) $q^{1}$ in $\chi_{1}\left(q^{1}\right)$ has been replaced by $i y$.

Equations (48) and (49) were derived by studying the behavior of asymptotic expansions across Stokes and anti-Stokes lines emerging from the two real or complex zeros, under the assumption that the other zeros have only a minor influence on the relevant asymptotic behavior. The equations reduce to their expected limiting values: When the transition points are sufficiently far apart, we have $A^{2} \gg 1$ in (48) and the usual WKB tunneling formula is obtained. When the barrier is parabolic (i.e., when $\beta, \Gamma=$ constant) the above equations reduce to the exact value ${ }^{11}$ known for Cartesian coordinates.

Several years ago Bell derived, on intuitive grounds for a parabolic barrier, an expression for the transmission coefficient ${ }^{12}$ which has found subsequent use in the literature. He made an excellent intuitive guess for $\kappa$, basing his arguments on the known limiting behavior of $\kappa$ at high energies $(\kappa=1)$, at very low energies $\left(\kappa=\right.$ simple WKB formula, $\left.A^{-2}\right)$, and at an energy just sufficient to surmount the barrier $\left(\kappa \cong \frac{1}{2}\right.$ according

${ }^{10}$ The equation for surmounting the barrier, in the form given here $[\mathrm{Eq}$. (49) ], presumes that the imaginary part of $B$ can be neglected (Ref. 4, p. 101). The imaginary part vanishes, of course, when $\Gamma$ is an even function of $q^{1}$.

"The exact solution for parabolic barriers may be found in $\mathrm{H}$. Jeffreys and B. Jeffreys, Methods of Mathematical Physics (Cambridge University Press, New York, 1962), p. 703, in Ref. 4, and in D. L. Hill and J. A. Wheeler, Phys. Rev. 89, 1102 (1953).

${ }_{12}$ R. P. Bell, Trans. Farday Soc. 55, 1 (1959). to the value found for the Eckart potential). His formula proves in fact to be identical with the exact result for a parabolic barrier in Cartesian coordinates.

\section{TRANSMISSION COEFFICIENTS IN ROTATION-FREE SYSTEMS}

We consider first two numerical computations of nonrotating collision complexes for the reaction $\mathrm{H}+$ $\mathrm{H}-\mathrm{H} \rightarrow \mathrm{H}-\mathrm{H}+\mathrm{H}$, made with the aid of electronic computers. A quantum-mechanical calculation of the probability that reactants in some initial state will form products on collision has been made by Mortensen and Pitzer. ${ }^{13}$ They made calculations for two internal coordinates as well as for several such coordinates taking into account bending vibrations of the collision complex in the latter case. When the $\mathrm{H}_{2}$ is in its lowest vibrational state numerical values of $\kappa$ can be largely explained by making a vibrationally adiabatic approximation: The observed $k$ 's agree reasonably well with one-dimensional Cartesian-coordinate $\kappa$ 's calculated by assuming that for all values of the reaction coordinate the vibrational motion can be described by a groundstate wavefunction. (Compare calculated and observed $\kappa$ 's in Table I, Ref. 7.) Some nonadiabaticity occurred, however, at least at higher translational energies. (Compare Table I, Ref. 13, $E=20, l=1$.) It would be useful to repeat the one-dimensional calculations ${ }^{7}$ taking into account the internal centrifugal effects. ${ }^{1}$

A classical-mechanical numerical calculation was made earlier by Wall, Hiller, and Mazur ${ }^{14}$ for the same system (two coordinates) but with a different potentialenergy surface. These authors found a surprising result: although their calculation was classical an initial vibrational energy of reactants equal to their zero-point energy lowered the initial translational energy needed for reaction by an amount equal to the difference of zero-point energies of the activated complex and the reactants. When the initial vibrational energy was three times the zero-point energy, the initial translational energy needed for reaction to occur was lowered by an amount which was three times this difference of zero-point energies.

In the writer's opinion a simple explanation of these results can be given by assuming that the motion was vibrationally adiabatic. For an adiabatic motion of a vibration whose frequency $\nu$ varies with position along some coordinate $q^{1}$ the action $J$ for the vibration is independent of $q^{115}$

$$
J=\oint p_{2} d q^{2}
$$

${ }^{13}$ E. M. Mortensen and K. S. Pitzer, Chem. Soc. (London) Spec. Publ. 16, 57 (1962)

${ }_{14}$ F. T. Wall, L. A. Hiller, Jr., and J. Mazur, J. Chem. Phys. 29, 255 (1958).

${ }_{15}$ This theorem is the classical adiabatic theorem, e.g., D. Ter Haar, Elements of Hamiltonian Mechanics (North-Holland Publishing Company, Amsterdam, 1961), p. $139 \mathrm{ff}$ 
where $q^{2}$ is the vibrational coordinate, $p_{2}$ its conjugate momentum, and integration at the given $q^{1}$-coordinate hypersurface is over a vibrational period. The vibrational energy equals $J \nu$, so that for a vibrationally adiabatic motion a portion of this energy, $J\left(\boldsymbol{\nu}^{\ddagger}-\boldsymbol{\nu}_{0}\right)$, must go into translational energy along $q^{1}$, where $\nu^{\ddagger}$ is the value of vibration frequency at the saddle point and $\nu_{0}$ is that for the reactants. In the two calculations of Wall et al., cited above, $J$ equaled $\frac{1}{2} h$ and $\frac{3}{2} h$, respectively. ${ }^{15 \mathrm{a}}$

It is possible, with the aid of analytical mechanics, to make a calculation of this tendency of $\lambda$ (quantum

${ }^{15 \mathrm{a}}$ Note added in proof: Further numerical calculations of trajectories in this system (with a different potential-energy surface) support this vibrational adiabaticity suggestion, not only for the rotation-free system but also, in contrast with earlier work, for the rotation-present one [M. Karplus, R. N. Porter, and R. D. Sharma (private communication)]. mechanics) or $J$ (classical mechanics) to be constant for the vibrational motion. Some calculations of this nature are now in progress. ${ }^{16}$

Wall, Hiller, and Mazur have also made numerical calculations on classical-mechanical transmission coefficients for the case of rotating systems. ${ }^{17}$ In this case the simple results described above for conversion of vibrational energy to energy useful for overcoming the barrier were apparently not obtained [see, however, Ref. 15(a) ]. It may be noted that in the activatedcomplex expression given by Eq. (15) vibrational adiabaticity was assumed only in the immediate vicinity of the activated complex, and not for all values of $q^{1}$ from region of reactants to that of products.

${ }^{16}$ R. A. Marcus (unpublished results).

${ }^{17}$ F. T. Wall, L. A. Hiller, Jr., and J. Mazur, J. Chem. Phys. 35, 1284 (1961).

\title{
Angular-Momentum Conditions for a Correlated Wavefunction
}

\author{
VINCENT MCKov \\ Department of Chemistry, California Institute of Technology, Pasadena, California*
}

(Received 21 April 1965)

\begin{abstract}
We obtain the conditions under which a correlation wavefunction containing pair correlation functions for the $2 p^{n}$ electrons of $\mathrm{C}\left({ }^{3} P,{ }^{1} D,{ }^{1} S\right), \mathrm{N}\left({ }^{4} S\right), \mathrm{O}\left({ }^{3} P\right)$, and $\mathrm{Ne}$ will be an eigenfunction of the orbital and spin angular-momentum operators. These pair functions contain quite general powers of electronic and interelectronic coordinates along with their variational parameters and should avoid the convergence difficulties of configuration interaction. In all these cases, except carbon which has just one pair of $2 p$ electrons, one cannot obtain such a pair function, $\hat{u}_{i j}{ }^{(1)}$, just by minimizing its pair energy, $\tilde{\epsilon}_{i j}{ }^{(1)}$, alone; i.e., minimizing the variational parameters of a $\hat{n}_{i j}{ }^{(1)}$. Minimization of one pair at a time corresponds to working with just a part of the correlation wavefunction which by itself cannot converge to the right symmetry state. One must minimize sums of pair energies by taking a correlation wavefunction containing enough pair functions so as to have the right symmetry. The sets of variational parameters in these different $\hat{u}_{i j}$ 's are not independent but are coupled. These symmetry requirements now make the determination of pair correlation functions quite a bit more difficult. In carbon $\left({ }^{3} P\right)$, these conditions do not apply, but the $a_{i j}\left({ }^{(1)}\right.$ of the $2 p^{2}$ electrons of the ${ }^{1} D$ multiplet must be made orthogonal to the unoccupied orbitals of the open shell if it is to contain general powers of the interelectronic coordinate. This is in addition to the required orthogonality to occupied orbitals. We also obtain a form of the correlation wavefunction for $2 p^{2}\left({ }^{1} S\right)$, a nonsingledeterminant Hartree-Fock (HF) state. This has not been previously known. We do this by applying the angular momentum stepdown operator to the correlation wavefunctions of multiplet states with single Slater determinant HF states and studying the resulting forms of the correlation wavefunction which, in turn, belong to some nonsingle-determinant states of these multiplets.
\end{abstract}

\section{INTRODUCTION}

A MANY-electron wavefunction contains a HartreeFock and a correlation part. This total wavefunction must be an eigenfunction of all operators that commute with the Hamiltonian; e.g., orbital $\left(L^{2}\right)$ and spin $\left(S^{2}\right)$ angular momentum of an atom. An approximate many-electron wavefunction will not, in general, be a simultaneous eigenfunction of all these commuting operators. Such wavefunctions are still useful for they may give good expectation values for

\footnotetext{
* Contribution No. 3234 .
}

many of these operators. Those operators, e.g., $L^{2}, S^{2}$, whose eigenvalues specify the symmetry of a state are particularly important. For example, in the variational determination of an approximate wavefunction, we must specify the symmetry properties of a trial function even before obtaining the expectation value of the Hamiltonian. This is clearly better than having to project out the desired symmetry component from a wavefunction of indefinite symmetry.

For an atomic system, it is quite simple to make the Hartree-Fock wavefunction an eigenfunction of $L^{2}$ and $S^{2}$, since its transformation properties depend 